\title{
Natural Decompositions of Perceived Transparency: Reply to Albert (2008)
}

\author{
Barton L. Anderson \\ University of Sydney and University of New South Wales
}

\author{
Manish Singh \\ Rutgers University
}

\author{
Judit O'Vari \\ University of Sydney and University of New South Wales
}

\begin{abstract}
In M. Singh and B. L. Anderson (2002), the authors proposed a model based on ratios of Michelson contrasts to explain how human observers quantitatively scale the perceived opacity of transparent surfaces. In subsequent work (B. L. Anderson, M. Singh, \& J. Meng, 2006), the authors found that this model failed to generalize to other contexts and replaced it with a new, more general model based on ratios of perceived contrasts. M. K. Albert's (2008) main experiment aimed to test the model the authors have previously rejected. The authors argue that M. K. Albert's experimental method was flawed and that his experiments did not test either the authors' original model or the authors' subsequent model that replaced it. M. K. Albert failed to provide any account of the data that the authors' model predicts, and he did not provide any theory to explain his own data. The authors conclude that the discrepancy between M. K. Albert's results and all models of transparency results from problems in the methods used in his experiments, not from the shortcomings of extant theory.
\end{abstract}

Keywords: transparency, scission, perceptual organization, lightness

An important goal of perceptual theory is to determine the natural decompositions of representational space, that is, to uncover the psychological dimensions that capture the variability of our experience. As the title of our original article suggests ("Toward a Perceptual Theory of Transparency"), our aim was to understand how human observers experience transparent surfaces. Previous theory was based on physical models of transparent surfaces, and that theory achieved some success at predicting the conditions under which transparency is perceived. But do physical model parameters capture the way psychological space is parameterized? If not, what parameterization best captures the dimensions along which transparent surfaces are experienced?

When viewing a transparency display such as in Figure 1, two dimensions of perceptual experience can be readily distinguished: Transparent surfaces appear to have a particular opacity $^{1}$ (or hiding power), and they appear to have a particular lightness (or, more generally, color). The most celebrated model of transparency at the time we wrote our original article (Singh \& Anderson, 2002) was Metelli's (1974a, 1974b, 1985), which contains two expressions that seem to map onto the two dimensions of our experience of transparent surfaces: one for the filter's transmittance and one for its reflectance. However,

Barton L. Anderson and Judit O'Vari, School of Psychology, University of Sydney, Sydney, Australia, and School of Psychology, University of New South Wales, Sydney, Australia; Manish Singh, Department of Psychology, Rutgers University.

Correspondence concerning this article should be addressed to Barton L. Anderson, School of Psychology, Brennan MacCallum Building (A18), University of Sydney, Sydney, New South Wales 2006, Australia. E-mail: barta@psych.usyd.edu.au our experiments revealed that these equations do not accurately describe how the opacity and lightness of transparent filters are perceived. Our data showed that both perceived opacity and lightness of transparent layers are strongly modulated by the mean luminance of the filter region. Specifically, we found that the opacity of the transparent layer appears to increase as the mean luminance of the filter region is increased (with its luminance range [maximum-minimum] kept fixed) and that the perceived lightness of the transparent filter is biased toward the mean luminance of the filter region. Thus, our main results showed that the perceived surface properties of a transparent layer are strongly dependent on the mean luminance of the filter region in a manner that is not captured by Metelli's (1974a, $1974 b, 1985)$ equations. We therefore proposed a simple way of modifying Metelli's (1974a, 1974b, 1985) equation for surface transmittance-using Michelson contrasts - that captured the dependence of perceived filter opacity on mean luminance. This simple modification provided an excellent account of our opacity data (Singh \& Anderson, 2002). We subsequently observed that this model failed to capture the perceived opacity of transparent surfaces in other contexts and developed a more general model based on perceived contrast (Anderson, Singh \& Meng, 2006).

In his critique, Albert (2008) presented data that he claimed contradicts our original and subsequent model of perceived transmittance. We contend that Albert's methods contain con-

\footnotetext{
${ }^{1}$ Throughout this article, we use the terms opacity and transmittance to refer to the same perceived dimension of a transparent surface, namely, the extent to which it appears to hide the underlying layer. Technically, the two are inversely related. For example, in Metelli's (1974a, 1974b, 1985) model, a filter's transmittance ( $\alpha$ in his model) was the fractional size of the missing sector in his episcotister; thus, $1-\alpha$ would be a measure of the filter's opacity.
} 


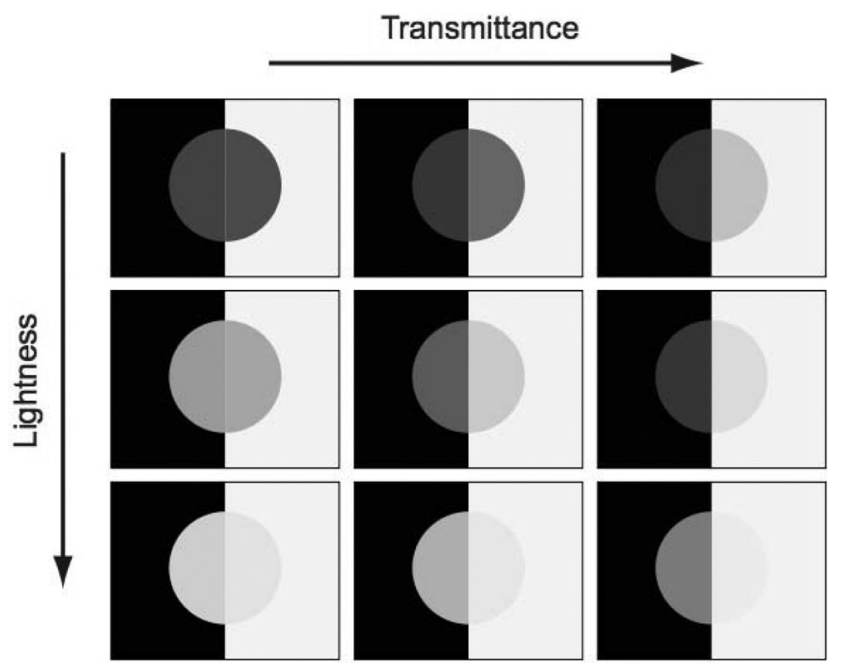

Figure 1. A demonstration to illustrate that perceived transparency is experienced as varying along two dimensions: transmittance (or opacity) and lightness (or color). The rows differ in the mean luminance and luminance range of their simulated backgrounds. The goal of a perceptual theory is to construct a theory that captures how human observers experience these two dimensions.

founds that preclude drawing any meaningful conclusions about the nature of the computations underlying transparency judgments. Moreover, even putting aside these confounds, Albert's experiment tested only the model that we had already rejected, not the model that replaced it. Our main arguments can be summarized as follows:

1. We believe that Albert's (2008) main experiment contains numerous methodological problems, the most significant being that observers could not independently manipulate the two main perceptual properties of filter. These problems are reflected in the fact that the data in his experiment are substantially less consistent than are data obtained in experiments from our or other laboratories (Singh \& Anderson, 2002; Robilotto, Khang, \& Zaidi, 2002; Robilotto \& Zaidi, 2004; Anderson et al., 2006). We argue that these data do not provide a basis for theory construction or evaluation.

2. Even if we were to ignore the methodological issues present in his experiments, Albert's (2008) data provide evidence against only the Michelson-contrast ratio version of our model. We have already reported the shortcomings of this version of the model (Anderson et al., 2006). His experiments do not provide a test of the perceived-contrast version of our model that replaced it - for the simple reason that his experiments did not independently measure perceived contrast in the transparency displays.

3. Albert (2008) asserts that his data provide evidence that our model is incorrect. To sustain his claim, Albert must demonstrate that his experiments measure the same perceptual property that was measured and modeled in our theory. Albert used a different experimental design, stimuli, and method of measurement, and he altered the task instructions of his experiment. The results he obtained are inconsistent with any existing theory or other data set in the field. We argue that Albert has not performed the necessary control experiment to demonstrate that his experiments measure the same perceptual properties as those embodied in our model, and hence, his experiment cannot be used to assess our model's veracity.

4. Albert's (2008) claim that our stimuli were not generic is incorrect. First, the center and surround in our match displays did differ in mean luminance. Second, his entire argument takes into account only the range of possible values that the parameters in Metelli's (1974a, 1974b, 1985) model can take (i.e., it inappropriately assumes a uniform distribution on that range). It ignores both the distribution of these parameters in naturally occurring forms of transparency, as well as the percept of transparency associated with these parameters. Our experiments focused on the paradigmatic cases of transparency that yield the strongest percept of scission into separate layers. Albert's experiments, on the other hand, introduce extreme differences in luminance between filter and background that may essentially block the form of image decomposition he is attempting to study.

In what follows, we provide more detailed arguments that expand on the points summarized above.

\section{Ratio-of-Contrasts Model}

We begin by considering the data that motivated our original model of perceived transmittance (Singh \& Anderson, 2002). Our stimuli were similar to Albert's (2008), but they had low-contrast sinusoidal texture patches placed against a fullcontrast sinusoidal surround for both the target (the display to be judged) and the match (the display adjusted by the observer to match the opacity the target filter; see Figure 2). The mean luminance of the target stimulus was fixed at the mean of the surround, and its luminance range varied across blocks. Within each block, the mean luminance of the match stimulus (the one observers adjusted) was varied from trial to trial. Observers were instructed to adjust the luminance range of the target pattern so that the opacity of the two filters appeared to be the same, even if they differed in perceived lightness. ${ }^{2}$ Metelli's (1974a, 1974b, 1985) model predicts that the perceived opacity of the filter should be independent of the mean luminance within the filter region and should depend only on the ratio of luminance $(\mathrm{L})$ ranges $\left(\mathrm{L}_{\operatorname{maxC}}-\mathrm{L}_{\operatorname{minC}}\right) /\left(\mathrm{L}_{\max S}-\mathrm{L}_{\operatorname{mins}}\right)$ between the center and the surround $(\max =$ maximum; $\min =$ minimum; $\mathrm{C}=$ center; $\mathrm{S}=$ surround). Contrary to this prediction, our results showed that (a) observers' matches of filter

\footnotetext{
2 These instructions were clarified with the hypothetical example of a red and a green filter, which can be perceived to be equally transmissive despite their obvious difference in color.
} 


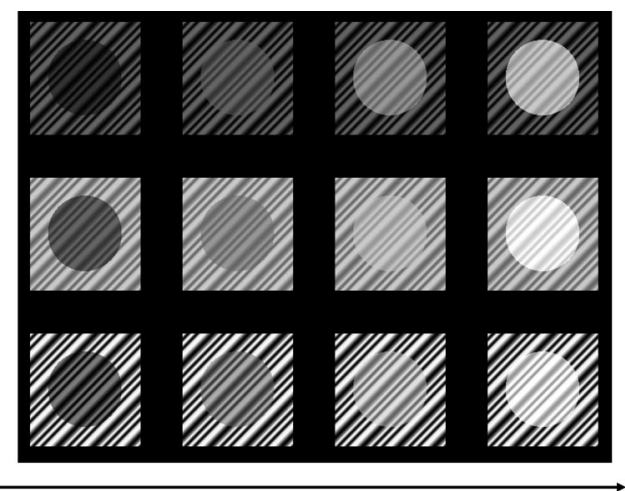

\section{Effective Luminance}

Figure 2. A demonstration of the influence of mean luminance on perceived opacity. In each of the three rows, the transmittance (as defined by Metelli's $\alpha$; 1974a, 1974b, 1985) is fixed, and the effective luminance is increased from left to right (i.e., the additive pedestal is increased from left to right, increasing mean luminance in the filter region). Note that these figures should be viewed with a linearized monitor to observe these effects.

transmittance exhibited a linear (scalar) increase in their luminance-range setting, with increasing mean luminance within the filter region of the matching stimulus, and (b) the slope of this linear dependence increased systematically with target filter transmittance.

This dependence can be experienced directly in Figure 2 (note that this figure must be viewed on a linearized monitor). In this figure, all of the targets within a row have the same multiplicative term (Metelli's $\alpha$; 1974a, 1974b, 1985), but the mean luminance of the central region is varied (corresponding to a change in the simulated lightness of the transparent layer). Note that the transmittance of the transparent layer appears to decrease as the mean luminance of the transparent region increases. This is true both for the full-contrast surround we used in our experiments (bottom row) and the two surrounds used in Albert's (2008) experiments (first and second rows). The results from our experiments confirmed this observation and revealed that the dependence of transmittance on mean luminance exhibits a simple, quantitative (linear) form.

To account for effect of mean luminance on transmittance matches, we suggested in our original article that Metelli's (1974a, $1975 b$, 1985) equation for transmittance — a ratio of luminance differences-should be replaced with a ratio of Michelson contrasts. There were numerous motivations for this proposal. First, there is a large body of evidence that suggests that the natural currency of the visual system is contrast, that is, some form of normalized luminance differences (i.e., luminance differences normalized by some function of mean luminance). Second, the use of Michelson contrast is one of the conceptually simplest ways to capture a dependence on mean luminance (because the normalization is proportional to mean luminance). Although our original model used a ratio of Michelson contrasts, there is nothing sacrosanct about this choice. It was merely a simple way to capture the dependence of transmittance matches we observed on the mean luminance of the target. It is important to note that this model provided a near-perfect account of our data. When observers' settings were replotted in terms of Michelson contrast, the data curves became independent of the filter region's mean luminance, demonstrating that observers were equating Michelson contrasts in the match and target displays (or some variable strongly correlated with Michelson contrast). In subsequent experiments, however, we found that ratios of Michelson contrast failed to capture perceived transmittance in other contexts, and we generalized this model to one involving a ratio of perceived contrasts (Anderson et al., 2006).

The other modeling decision we had to make was incorporating the fact that the perceived transmittance of a transparent surface is inherently a relative judgment. For simplicity, consider displays in which the mean luminance of the center and surround are the same. In such contexts, it is a perceptual fact that the center will appear transparent only when its luminance range is smaller than the surround. Moreover, the transmittance of the transparent layer-its hiding power-is determined by the extent to which the luminance range of the surround is changed in the region of transparency. More precisely, the transmittance of a transparent layer is determined by some measure of how it perturbs the visibility of the underlying layer. (To determine the hiding power of surface, one must have some idea of the surface that it is hiding.) Therefore, the question that must be addressed is as follows: What is the appropriate relative measure that accounts for the perception of transmittance? Our answer to this question was borrowed from Metelli (1974a, 1974b, 1985, and, by analogy, from Wallach's ratio rule in lightness perception). Metelli's (1974a, 1974b, 1985) episcotister model proposed that transmittance $(\alpha)$ is determined by the ratio of luminance differences of the filter region to that in the background. We therefore modified this proposal in the simplest way possible to fit our data: We proposed that the appropriate relative measure takes the form of a ratio. But rather than a ratio of luminance differences, we proposed a ratio of Michelson contrasts. These are the core elements of our original model, and the main insights that shaped it as well as our subsequent generalization based on the ratio of perceived contrasts.

Note that the contrast of the surround plays the role of an anchor in our model; that is, it defines a region in plain view, ${ }^{3}$ and is a divisive normalization factor that is used to compute the transmittance of the transparent layer. In our original experiment, this divisive factor was the same in the target and match pattern, so this experiment did not explicitly test this aspect of our model. However, in Anderson et al. (2006), we did precisely this. Our results showed that when the background contrasts differed by a factor of 2.2 in the target and match displays, local contrast matches of the filter region completely failed to predict perceived transmittance, whereas the ratio of filter-to-background perceived contrasts predicted transmittance matches almost perfectly. It is noteworthy that Albert (2008) fails to address these data in any substantive way. We argue that this feature of our model-the presence of some

\footnotetext{
${ }^{3}$ There is in fact a deeper problem the visual system faces, which is that images do not come prepackaged into filter regions and backgrounds-as is implicitly assumed in many classical treatments. A large part of solving the transparency problem consists precisely in determining which image regions correspond to transparency versus surfaces in plain view. This is when the notion of anchoring becomes critical (e.g., Anderson, 2003; Anderson et al., 2006; Singh \& Anderson, 2006).
} 
divisive normalization by regions in plain view-is essential. It is needed not only to account for our data but also to provide a unified account of the other data sets from other labs as well.

\section{Additional Evidence for the Role of Contrast in Transparency Judgments}

Shortly after our article demonstrating the role of contrast in transparency perception, articles from Zaidi's laboratory (Robilotto et al., 2002; Robilotto \& Zaidi, 2004) provided additional evidence for the importance of contrast in perceived transparency. The main task variation introduced by these authors was to provide minimal instructions to their observers, who were told only to match filters for their perceived transparency. Their expressed goal was to see what aspects of the displays observers equated under these instructions and to determine whether observers could "carry out the task in a consistent and meaningful manner" (Robilotto \& Zaidi, 2004, p. 190). In their experiments, observers adjusted either the inner transmittance or the reflectivity of a simulated transparent filter to match a target filter. ${ }^{4}$ Using this paradigm, Robilotto and Zaidi (2004) found that observers' transparency matches corresponded closely to the transmittance of the simulated filters. Albert (2008) never addressed this highly pertinent result, so it is unclear what he intended by the claim that "there might be no such thing as perceived transmittance" (p. 1139).

For present purposes, the critical finding in Robilotto et al.'s (2002) work is that transparency matches were well predicted by perceived contrast: a control experiment revealed that observers made essentially identical matches when instructed to match perceived contrast instead of perceived transparency. Hence perceived contrast serves as a sensory determinant of perceived transparency under the intentionally unconstrained instruction set. As with our subsequent work (Anderson et al., 2006), Robilotto and colleagues' (2002) experiments showed that Michelson contrast did not capture perceived contrast in general. For the texture patterns used in their studies, Robilotto et al. (2002) found that no standard measure of contrast captured perceived contrast. Nonetheless, they consistently found that perceived contrast predicted observers' transparency matches.

A major point of convergence in our findings and those of Robilotto and colleagues is, thus, the critical role played by perceived contrast in transparency judgments. However, there appears to be-at least on the surface-one point of difference between our results and those of Robilotto et al. (2002) and Robilotto and Zaidi (2004). Their results showed that observers made essentially the same matches when equating perceived transparency and equating perceived contrast within the central texture patch. This seems to suggest that perceived transparency is determined by the local contrast within the filter region, rather than the ratio of contrasts - center to surround. On the other hand, our results clearly showed that local contrast matches do not predict perceived transmittance, whereas contrast ratios do (Anderson et al., 2006). Based on this apparent difference, Albert (2008) asserted that the work from Zaidi's lab provides evidence against our model, because he claimed that their observers equated "perceived absolute contrast" (p. 1131). It is readily demonstrated, however, that there is in fact no inconsistency between our results and those of Robilotto et al. (2002) and Robilotto and Zaidi's (2004) and that their results, like ours, are consistent with our model.
Robilotto and colleagues $(2002,2004)$ used two tasks: a transparency matching task and a contrast matching task. In the transparency matching experiment, observers adjusted one of two simulated filter properties (either inner transmittance or reflectivity) to equate the perceived transparency of the two filters. In the contrast matching experiment, observers adjusted the same two parameters to match the perceived contrast of the target. In the contrast matching experiment, however, the backgrounds (in both the target and match display) were rotated relative to the centers to disrupt the percept of transparency. In both experiments, only one independent simulated property was varied at a time, something that is not true of Albert's (2008) experiments (see below). Robilotto and colleagues (2002) obtained highly consistent-and essentially identical-results in their transparency and contrast matching experiments, suggesting that observers were equating perceived contrast to achieve both matches. The question is whether these results reveal that the observers are equating absolute contrast rather than contrast ratios, as Albert asserted.

It has been well documented that the perceived contrast of a target is influenced by the contrast of its surround (e.g., Chubb, Sperling, \& Solomon, 1989; Solomon, Sperling \& Chubb, 1993; D'Zmura \& Singer, 1999). This influence is usually modeled as a form of normalization by the surround contrast. The extent of this normalization depends on factors such as the similarity between the center and the surround (in terms of orientation, phase, or other manipulated variables). Thus, the normalizing effect of the surround can be weighted

$$
C C /(k * S C),
$$

where $k$ is a parameter that determines the strength of the normalization, $C C$ is center contrast, and $S C$ is surround contrast. ${ }^{5}$ If we assume that observers simply match perceived contrast in Robilotto \& Zaidi's (2004) contrast matching experiment, the observer would thus make contrast settings so that the perceived normalized contrast is equated in the target and match. That means equating

$$
T F C /(k * T B C)=\operatorname{MFC} /(k * M B C),
$$

where $T F C$ is target filter contrast, $T B C$ is the target background contrast, $M F C$ is the matching filter contrast, and $M B C$ is matching background contrast. Under the assumption that the effect of surround rotation in the match and target displays caused equal reduction in the strength of the contrast normalization, the two $\mathrm{ks}$ in Equation 2 simply cancel out, generating exactly the same matches as those predicted by a simple contrast ratio model (i.e., one that does not involve any weighting parameter $k$ ). If the reduction in normalization in the two displays were only approximately equal, the matches based on perceived normalized contrasts would be approximately equal to those based on contrast ratios. Thus, there is nothing in Robilotto and Zaidi's (2004) data that contradicts our model.

\footnotetext{
${ }^{4}$ Inner transmittance and reflectivity are the parameters of a physical model of neutral density filters (Wyszecki \& Stiles, 1982). They do not correspond respectively to the overall transmittance and the reflectance of a filter. Indeed, both filter transmittance and reflectance are functions of both inner transmittance and reflectivity (see Robilotto et al., 2002 for details).

5 The arguments developed here are independent of the specific contrast measure used to characterize local contrast (i.e., Michelson, RMS, etc.).
} 
The preceding analysis reveals the relationship between a contrast ratio model of perceived transmittance and a model in which transmittance matches are made by matching the perceived contrast of the transparent regions directly. Albert (2008) interpreted Robilotto and Zaidi (2004)'s results to mean that perceived transparency "corresponds closely to the perceived absolute contrast of the filter region (p. 1131)." But this interpretation fails to appreciate that the large literature on contrast induction demonstrates that this is simply not possible. In the contrast ratio model we proposed, the transmittance of the transparent layer is normalized by the full surround contrast. In a model in which perceived contrasts are matched, the surround contrast still has a modulatory effect on the contrast of the center, but to a greater or lesser extent (as the contrast literature has shown). Thus, the issue of whether absolute contrast or contrast ratios are used to determine perceived transmittance is largely an argument about the extent to which the surround affects transparency judgments, not whether it is used. As we noted above, one can determine the hiding power of a transparent surface from an image only if one has some idea of the surface that it is hiding; any estimate of transmittance must therefore involve some comparison with surfaces seen in plain view.

\section{Albert's (2008) Experiments}

Albert's (2008) transmittance matching experiment (Experiment 1) required observers to match displays in which the mean luminance and contrast of the centers and the surrounds differed in both the target and match display. In Albert's displays, the background of the match pattern was 3 times as bright as that of the target, and the Michelson contrast of the target background was also approximately 3 times as high as that of the match. There are a host of differences between Albert's experiments and ours, so it is difficult to know from his report what was responsible for the noisy pattern of results he obtained. In order to attempt to determine what observers might have been doing that led to his pattern of results, we recreated his experiments. We believe that there are numerous problems with his experimental design that led to the noisy data that he presents as evidence against our model. Due to space restrictions, we focus on the following points: the flaws in Albert's method; the pattern of overestimation of transmittance in his data, which is readily explained; his claim of implausibility of our ratio model; the failure of his experiments to provide a test of our current model; and his failure to perform appropriate control experiments to demonstrate that his experiment measured the same variables as those expressed in our model.

1. Albert's (2008) experimental design required observers to adjust a match pattern to appear identical in transmittance to that of the test pattern. Albert chose to vary the simulated transmittance ( $\alpha$, the multiplicative term that varies between 0 and 1) while its effective luminance (the additive term) was clamped at a constant value. This parameterization of this match pattern has the undesirable consequence that when the simulated transmittance of the match is increased, its simulated (and, more importantly, perceived) lightness also increases (see Figure 3). Thus, if observers reduce the transmittance of the match relative to the target, it also appears to become darker than the target; if they increase it, it becomes lighter than the target. When our naïve participants attempted to perform this matching task using this parameterization, they reported that they found themselves adopting a strategy of minimizing the overall difference in
Effective Luminance

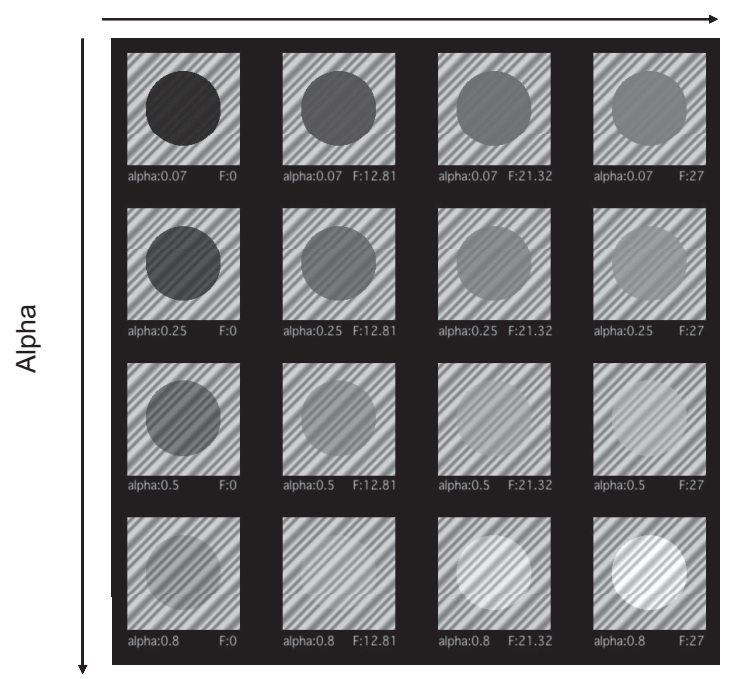

Figure 3. A figure demonstrating that Albert's (2008) match adjustment pattern conflated the perceived lightness and opacity of the transparent region. In Albert's experiments, observers adjusted the simulated alpha, while the effective luminance (the additive term) was held fixed. Each column depicts a fixed effective luminance and varying alpha. In Metelli's (1974a, 1974b, 1985) model, this implies that the simulated transmittance and simulated reflectance of the transparent layer both appeared to change as observers attempted to match the transmittance of the target pattern. It is likely that observers merely attempted to minimize the overall appearance of the two filters with this adjustment paradigm. Note that these figures should be viewed with a linearized monitor. $\mathrm{F}=$ effective luminance.

appearance between match and target filter, rather than matching transmittance (see also Point 5, below). Thus, there is no guarantee that Albert's observers were performing transmittance matches at all. Instead, it is likely that they were simply minimizing some measure of overall difference between match and test displays (based on both filter transmittance and lightness). This interpretation is in fact supported by Albert's data.

2. The one relatively consistent trend in Albert's (2008) data is that observers tend to overestimate the perceived transmittance of the test filter (as predicted either by the Michelson contrast version of our model or by Metelli's, 1974a, 1974b, 1985, model). There are two possible factors that may have led to this pattern of results. First, the surround of his target pattern had a minimum luminance of 0 , which means that the Michelson contrast of the surround would be automatically 1 (i.e., full contrast) irrespective of the choice of maximum luminance. Given the restricted range of luminance values from black to mid-grey, it is unlikely that observers perceive such surrounds as having a full contrast; in other words, they would systematically underestimate the contrast of the surround. This, in turn, would lead observers to overestimate the transmittance of the filter in the target, which is consistent with the general pattern in his data. Consistent with this interpretation, Robilotto and Zaidi (2004) found that lowering the luminance of their surrounds had a nearly identical effect in their data as lowering the contrast of the surround. This provides strong evidence that the contrast of Albert's target surround was not perceived as full contrast. 
Second, Albert's (2008) match filter on the (light) background appears darker than the corresponding target filter (at least for effective luminances greater than zero). Thus, if observers decreased the transmittance of the simulated filter to equate their Michelson contrast ratios, the match filter appeared much darker than the target (see Figure 2). It is likely that observers were thus strongly biased to match perceived brightness, rather than just perceived transmittance, when performing their matches. If true, Albert's data do not provide an uncontaminated measure of perceived transmittance.

One of the most important issues in designing a psychophysical experiment is to provide a method that measures the perceptual property of interest. This is particularly critical in a method of adjustment, where the knob that one provides observers to adjust provides information about the dimension of the display that observers are attempting to judge. Albert (2008) criticized our matching method because our observers were only allowed to adjust the luminance range of the match pattern while its mean luminance was held constant. We believe that this is precisely the strength of our method because the only variable that appears to change in our matching pattern is its perceived transmittance, the dimension observers are attempting to judge. ${ }^{6}$ This factor may have also contributed to the relatively variable pattern of results that we observed when observers performed lightness matches of transparent filters in our original study (Singh \& Anderson, 2002). In our lightness-matching experiment, observers varied the mean luminance of our target filter (while its luminance range was kept fixed) to match the perceived lightness of the match filter. This parameterization caused both the lightness and opacity of the filter to covary, which may have contributed to the relatively noisy pattern of data within and across observers in this experiment. Thus, the problem of designing an appropriate measurement tool may have played a significant role in our lightness experiment as well.

3. Albert (2008) asserted that our contrast ratio model is inherently implausible because it predicts that all filters with a simulated reflectance of zero should appear equally transmissive. This is indeed a prediction of our model-as long as the filter preserves perceived contrast, not just Michelson contrast-and we do not think that this makes it implausible. Filters with a simulated reflectance of zero (which are not physically realizable within Metelli's (1974a, 1974b, 1985) model; see the section on genericity, below) would be photometrically indistinguishable from shadows - that is, changes in illumination - that are contrast preserving. In our view, the visual system does not treat shadows as having a greater degree of opacity than a directly illuminated region; rather, shadowed and nonshadowed regions are both seen as being in plain view, but under different illuminants. In other words, shadows appear to vary along a light-dark scale, not along an opacity scale. Our (psychological) model of perceived transmittance naturally captures this property of human experience, whereas physical models (such as Metelli's, 1974a, $1974 b, 1985)$ do not. In this respect, it is noteworthy that this condition in Albert's experiment (i.e., $F=0$ ) exhibits the highest variability across participants, suggesting that observers had to adopt a different strategy in this regime.

4. Albert (2008) performed two control experiments in an attempt to assess the ratio-of-perceived contrasts model. Unfortunately, neither experiment provides data that bear on our model. His Experiment 2 required observers to equate the center-tosurround contrast ratios in the target and match displays. It is not clear what this experiment was designed to test. We did not claim that observers explicitly judge contrast ratios; rather, we proposed that the visual system uses contrast ratios between a region in plain view and a transparent region to compute the perceived transmittance of a filter. By analogy, it has been shown that in some contexts, the lightness of two surface patches under different illuminants is perceived to be the same when their luminance ratios are equal. Observers in such experiments are not instructed to explicitly match luminance ratios; they are instructed to match (or otherwise judge) the perceived lightness of a target.

Nevertheless, it appears from his data that observers are in fact able to match contrast ratios. Albert (2008) claims that the variability of the data in this experiment "especially between observers (p. 1134)" provides evidence against the plausibility that contrast ratios are used in making transparency judgments. Nothing in Albert's data in fact supports this assertion; the data from his Experiment 2 were actually much less variable across observers than were data from his first experiment. The only exception to this was the data for which Albert himself served as the observer (observer S5). It would therefore appear that observers (other than Albert) have a much clearer idea of what it means to match contrast ratios in his experiments than to match the "percentage of light transmitted from a background."

Albert's (2008) second control experiment (Experiment 3) provided only half of the data needed to assess our ratio of perceived contrasts model. It obtained measurements of perceived contrast of the center, but not of the surround. A ratio of perceived contrasts model clearly cannot be tested without both sets of measurements. This omission is particularly important given that the surround of his target in Experiment 1 had a minimum luminance of 0 , hence automatically a Michelson contrast of 1 irrespective of the choice of maximum luminance (see his Figure B1; surround on the right side). As noted above, it is almost certain that observers do not perceive the contrast of this surround as full contrast, which means Albert has not tested our perceived contrast ratio model.

5. Albert's (2008) argument that his data demonstrated that our model is incorrect hinges on his ability to demonstrate that the perceptual variable he measured in his experiment is the same that we have (successfully) modeled in our data. This is the typical function of a control experiment (e.g., one that would involve the same instructions, parameterization, or range of stimulus parameters as our experiments), but no such experiment was performed. Instead, Albert simply noted that our model fails to capture the data in an experiment in which he simultaneously changed the method, the task, and the parameterization of his measurement tool (the adjustment method). All existing models fail to capture his data, and his data are inconsistent with all other published data. Without an appropriate control experiment, it is impossible to determine the cause of this difference.

The problem of determining what perceptual variables form the basis of observers' responses is particularly significant in trans-

\footnotetext{
${ }^{6}$ Albert (2008) also contends that we may have obtained our pattern of results because observers could only adjust contrast. This argument is clearly fallacious. Our observers in fact adjusted the luminance range within the region of transparency (while its mean luminance was fixed). By Albert's reasoning, it should be impossible for our results to exhibit any dependence of opacity matches on mean luminance. But this is precisely what our results showed.
} 
parency research because they are attempting to make judgments in image regions that appear to contain more than one material layer. It has been shown, for example, that scission can induce dramatic shifts in perceived lightness (Anderson, 1997, 1999; Anderson \& Winawer, 2005, 2008). However, the direction of the lightness shift depends critically on which layer the observer is asked to report. This means that experiments on perceived lightness shifts in conditions of transparency could yield exactly opposite results if a change in experimental design caused observers to base their responses on different layers. Similar issues may also arise in transmittance judgments, a point Albert (2008) himself raised in conjunction with his discussion of Robilotto and Zaidi's (2004) experiments. Given these ambiguities, all that Albert can logically deduce from his (noisy) results is that his design generated different results than those that have been observed previously in transparency experiments. His argument that our model is incorrect cannot be justified on the basis of the mere discrepancy between his results and our model because his experiments lack the appropriate control to determine whether observers are basing their responses on the same perceptual attributes as those captured in our model.

In sum, there are a number of problems with Albert's (2008) experiments and model predictions that limit their value in theory evaluation and construction. The high variability of his data in Experiment 1, particularly between observers, suggests there are significant design problems that prevent observers from performing his task in a consistent manner. There may also be significant problems with his choice of stimuli, because previous studies did not use stimuli with such extreme differences in mean luminance between the center and the surround. Indeed, recent work suggests that large luminance differences between filter and background regions may effectively block scission into separate layers in transparency (see below). Finally, Albert never performed a control experiment to determine whether his experimental design measures the same perceptual dimension that led to the formation structure of our model. Hence, his claims that he has demonstrated that our model is incorrect cannot be justified on the basis of the data that he presents. It is difficult to know what factor, or combination of factors, led to the inconsistent pattern of results obtained in Albert's experiments. Whatever their cause(s), it is nonetheless descriptively true that his experiments were much less internally consistent than any other studies that have been undertaken in this field. Albert has provided no explanation of this fact.

\section{Genericity and the Conditions for Scission}

One of Albert's (2008) critiques of our work is that our experiments and model are special cases, and hence do not represent the generic conditions of transparency. There are a number of problems with this argument. First, the assertion that our opacity matching experiment only used stimuli in which the filter and background had equal mean luminance is factually incorrect. The center and surround in our match displays differed substantially in mean luminance. (Performing the opacity-matching task of course required observers to estimate opacity in both the target and match.) Second, Albert's entire genericity argument only takes into account the possible parameter values that a physical model of transparency (such as Metelli's, 1974a, 1974b, 1985) can take. In other words, the argument inappropriately assumes a uniform distribution on the range of parameter values. It ignores both the distribution of these parameters in naturally occurring forms of transparency, as well as the percept of transparency associated with these parameter values.

The "generic" conditions for transparency that the visual system confronts must be justified by the distribution of naturally occurring forms of transparency, not by the range of parameters that are mathematically possible. Indeed, one of our motivations for exploring transparency induced by continuous media (Anderson et al., 2006) is that this seems to be the most common form of transparency in natural scenes. The sharp-edge filters that have dominated the perceived transparency literature are largely manufactured contrivances; they play little or no role in most natural scenes. Similarly, considerations of naturally occurring forms of transparency also suggest that the condition in which the additive component of the filter is zero $(F=0)$ would not just be nongeneric in Metelli's (1974a, 1974b, 1985) model, it is physically unrealizable because Metelli's (1974a, 1974b, 1985) model assumes that all of the surfaces are equally illuminated, and even the blackest material reflects $\sim 5 \%$ of the light striking it.

Moreover, recent work has shown that scission is most likely to occur in center-surround displays when the chromatic and achromatic contrast between the center and the surround is low (Ekroll, Faul, \& Niederee, 2004; Faul, Ekroll \& Wendt, 2008). Albert's (2008) failure to observe reliable judgments of transmittance may arise because the contrast between the center and the surround was high in both his target and match pattern, which may have reduced or interfered with the scission of the target region, and may have effectively suppressed the form of image decomposition he was attempting to study. If, in our experiments, we did study special cases, it is because such cases most strongly induce scission into separate layers, which is necessary for a meaningful study into transparency perception.

\section{Conclusions}

We have argued that Albert's (2008) claim that his experiments demonstrate that our model is incorrect is unjustified. We believe that the core elements of our model are critical ingredients for any model of transparent surfaces and media in conditions of overlay. The goal of our experiments was to measure the dimensions that underlie our psychological representation of transparent surfaces and media. There is a long history in perceptual theorizing of treating generative physical models as serving double duty: as descriptions of the physics that give rise to images, and as implicit models of what the visual system must be computing. We believe that this can be misleading. Psychological theories of perception need to uncover the variables that best capture the dimensions along which our perceptual experience varies - which may or may not correspond to the variables in a physical model. Both our and other researchers' subsequent data have revealed that relative contrast plays a critical role in the perception of transmittance (or perceived transparency) of a surface. If observers are simply asked to match the perceived opacity in displays that elicit a clear decomposition into separate layers and are given a matching pattern in which opacity is the only perceptual dimension that varies, they find this task to be easy and natural, and the data obtained in such experiments is highly consistent both within and across observers. This was not the case in Albert's experiments.

We have little doubt that the model that we have advocated will undergo significant revision as more is learned about the set of 
factors that modulate the perceived lightness and opacity of transparent surfaces. However, we believe that the core insights that shaped our model will be incorporated into future successful models of the perception of transparency. There appear to be two primary dimensions that capture the psychological experience of transparent surfaces: opacity and lightness (or, more generally, color). Physical models such as Metelli's (1974a, 1974b, 1985) also appear to embody the same two dimensions, which is probably why the model garnered so much attention as a candidate model of perception. The problem is that the physical decomposition embodied in Metelli's (1974a, 1974b, 1985) model does not accord with the perceptual experience of these dimensions - which is why we developed a new model to replace it.

Albert (2008) claims he has shown our model to be incorrect without providing any understanding of the data for which the model has provided excellent accounts. We believe that the logic that has led him to this conclusion is flawed. Our model was motivated by the observed dependence of transmittance and lightness matches on mean luminance within the filter region. Even if we were to ignore the methodological problems discussed above, Albert's data merely indicate that mean luminance can influence transmittance matches in a manner beyond that captured in computations of contrast. It is currently impossible to determine whether such dependencies will replace those captured in our contrast ratio models or will merely provide an additional factor that contributes to the perception of transparency.

Finally, Albert (2008) offered no theoretical account of how transparent surfaces are perceived, nor does he attempt to model or provide any theoretical insight into his own data. Instead, he merely asserts that, for his task, our model fails to explain his data. All existing models fail to explain his data, so it is unclear why he is content with discussing his data only in the context of our model. Given his evidence, we are inclined to believe that this putative failure is not due to the shortcomings of extant theory, but rather, to the methods used in his experiments.

\section{References}

Albert, M. K. (2008). The role of contrast in the perception of achromatic transparency: Comment on Singh \& Anderson (2002) and Anderson (2003). Psychological Review, 115, 1127-1143.

Anderson, B. L. (1997). A theory of illusory lightness and transparency in monocular and binocular images: The role of contour junctions. Perception, 26, 419-453.
Anderson, B. L. (1999). Stereoscopic surface perception. Neuron, 26, 919-928.

Anderson, B. L. (2003). The role of occlusion in the perception of depth, lightness, and opacity. Psychological Review, 110, 762-784.

Anderson, B. L., Singh, M., \& Meng, J. (2006). The perceived transmittance of inhomogeneous surfaces and media. Vision Research, 46, 1982-1995.

Anderson, B. L., \& Winawer, J. (2005, March 3). Image segmentation and lightness perception. Nature, 434, 79-83.

Anderson, B. L., \& Winawer, J. (2008). Layered image representations and the computation of surface lightness. Journal of Vision, 8, 1-22. doi: 10.1167/8.7.18.

Chubb, C., Sperling, G., \& Solomon, J. (1989). Texture interactions determine perceived contrast. Proceedings of the National Academy of Science, USA, 86, 9631-9635.

D'Zmura, M., \& Singer, B. (1999). Contrast gain control. In L. T. Sharpe \& K. R. Gegenfurtner (Eds.), Color vision: From genes to perception (pp. 369-385). Cambridge, England: Cambridge University Press.

Ekroll, V., Faul, F., \& Niederee, R. (2004). The peculiar nature of simultaneous contrast in uniform surrounds. Vision Research, 44, 1765-1786.

Faul, F., Ekroll, V., \& Wendt, G. (2008). Color appearance: The limited role of chromatic surround variance in the "gamut expansion effect." Journal of Vision, 8(3), 1-20.

Metelli, F. (1974a). Achromatic color conditions in the perception of transparency. In R. B. MacLeod \& H. L. Pick (Eds.), Perception: Essays in honor of J. J. Gibson (pp. 95-116). Ithaca, NY: Cornell University Press.

Metelli, F. (1974b, April). The perception of transparency. Scientific American, 230, 90-98.

Metelli, F. (1985). Stimulation and perception of transparency. Psychological Research, 47, 185-202.

Robilotto, R., Khang, B., \& Zaidi, Q. (2002). Sensory and physical determinants of perceived achromatic transparency. Journal of Vision, 2(5), $388-403$.

Robilotto, R., \& Zaidi, Q. (2004). Perceived transparency of neutral density filters across dissimilar backgrounds. Journal of Vision, 4(3), 183-195.

Singh, M., \& Anderson, B. L. (2002). Toward a perceptual theory of transparency. Psychological Review, 109, 492-519.

Singh, M., \& Anderson, B. L. (2006). Photometric determinants of perceived transparency. Vision Research, 46, 879-894.

Solomon, J., Sperling, G., \& Chubb, C. (1993). The lateral inhibition of perceived contrast is indifferent to on-center/off-center segregation, but specific to orientation. Vision Research, 33, 2671-2683.

Wyszecki, G., \& Stiles, W. S. (1982). Color science: Concepts and methods, quantitative data and formulae (2nd ed.). New York: Wiley.

Received August 23, 2007

Revision received July 29, 2008

Accepted July 30, 2008

\section{Postscript: Qualifying and Quantifying Constraints on Perceived Transparency}

\author{
Barton L. Anderson \\ University of Sydney

Manish Singh
Rutgers University
Judit O'Vari
University of Sydney

Contrary to Albert's (2008) claims, the results of previous studies do not favor a perceived contrast model over a ratio-of- perceived-contrasts model (see Points $1-3$ below and our main response). Realizing that a simple perceived contrast model leads to predictions that violate "common sense," Albert postulated a division of the continuous dimension of perceived opacity into a small number of distinct qualitative regimes of transparency, arguing that subjects will only match opacity within the same regime. Questions of parsimony aside, critical issues concerning how the model actually works are left unaddressed: How does the visual system decide to which qualitative regime a display belongs (e.g., highly transparent vs. medium transparent)? Albert simply listed filter-to-background relative properties (which we have previously articulated as being crucial, including ratio-of-perceived contrast) without specifying how such variables determine the 
categories of filters he proposed. According to his model, a subject would first adjust the match display so the two filters are in the same qualitative regime and would then match perceived contrast. However, once the two filters have been brought into the same regime, there is no guarantee that a perceived contrast match will be available (especially if the two display backgrounds differ strongly in contrast). It is completely unspecified what his model predicts in such conditions.

Regarding the data from Experiment 1 in Anderson, Singh, and Meng (2006), Albert (2008) conceded that a simple contrast matching strategy fails to account for the most transmissive settings observers made to the contrast peaks of our stimuli-which differed in (Michelson) contrast by a factor over 2 but which were matched nearly identically. Nonetheless, Albert asserted that observers primarily match perceived contrast near the opaque range of the scale and argued that data from this experiment are highly inconsistent with our model. Both claims are demonstrably incorrect. Experiment 5 of Anderson et al. (2006) showed that transmittance settings near the contrast troughs differed by a factor of 2 ( $20 \%$ versus $40 \%$ ) when different match patterns were used. Consistent with our ratio-of-perceived contrast model, the data from these two experiments become indistinguishable when normalized by their background contrasts (see Figure P1 Panel A). A simple perceived contrast matching model fails to account for these data

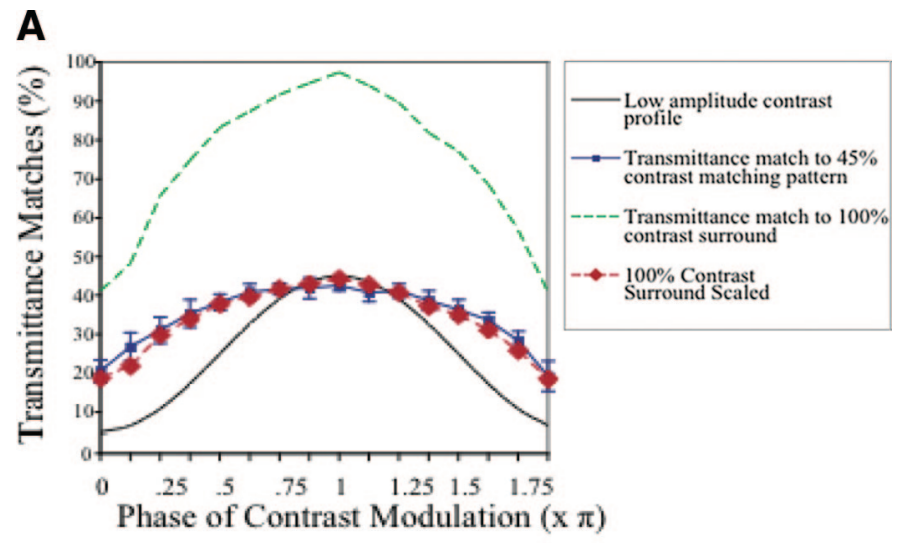

B

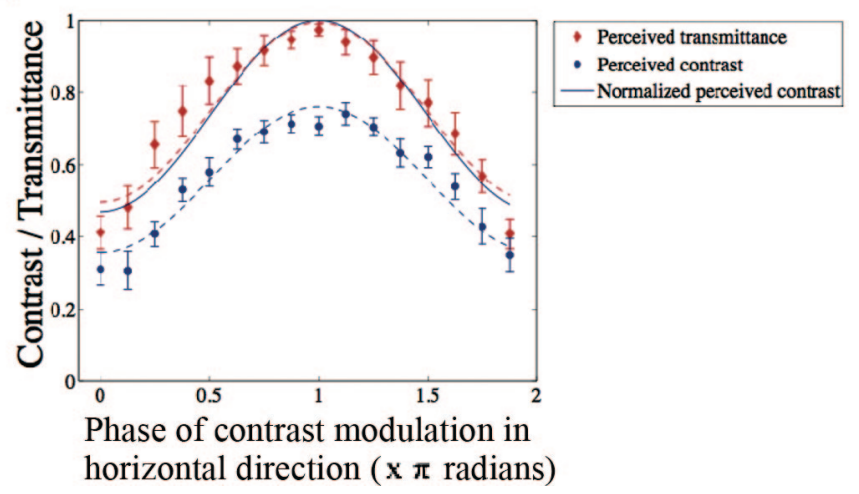

Figure P1. Adapted from "The Perceived Transmittance of Inhomogeneous Surfaces and Media," by B. L. Anderson, M. Singh, and J. Meng, 2006, Vision Research, 46, pp. 1991 \& 1993. Copyright 2006 by Elsevier. Transmittance matches using $45 \%$ contrast matching pattern. Data from two experiments demonstrating that observers do not simply equate perceived contrast in any regions of the transparency displays in Anderson et al. (2006). Error bars are 95\% confidence intervals. A: Data from Experiment 5 compared with data from Experiment 1. In both experiments, observers adjusted the luminance range of a central patch to match the perceived opacity of a contrast modulated random dot pattern. In Experiment 1, the contrast of the surround was maximal for the monitor (95\%); in Experiment 5, the surround contrast was 45\%. Albert's (2008) model indicates that observer's resort to matching perceived contrast in the near-opaque regions of the display, which occur on the far right and left of the graph. Matches in the regions differed by a factor of 2 (20\% vs. $40 \%)$, contradicting his model. However, the matches from these two experiments become indistinguishable when normalized by a factor that places them on the same scale, as predicted by our ratio-of-perceived contrast model. B: Observers' transmittance settings are plotted against their perceived contrast settings for the same lowcontrast modulated pattern. Again, perceived contrast is not equal for any regions of the curves. But, when the perceived contrast data are normalized by their maximum (i.e., the transmittance anchor in our model), the transformed perceived contrast data (solid blue line) are indistinguishable from observer's transmittance matches (dashed upper red line). 
in any region of the display. In Experiment 6, observers matched the perceived contrast of our low-contrast target using the same match display as for their transmittance matches. Here, again, observer's transmittance matches are never predicted by their perceived contrast matches (see Figure P1 Panel B). However, when the perceived contrast data are scaled by their transmittance anchor (their highest perceived contrast setting) they become indistinguishable from observer's transmittance matches (see the curve fits in Figure P1 Panel B). In other words, the perceived contrast and transmittance matches differ by a fixed scaling factor over the entire curve, precisely as the ratio-of-perceived contrast model predicts. Albert's model completely failed to account for this finding.

Albert (2008) contended that the identity of observers' transmittance matches to the contrast troughs (nearly opaque regions) of our high-contrast stimulus (HCS) and low-contrast stimulus (LCS) is highly inconsistent with our model. In fact, the opposite is true: These data are perfectly consistent with ratio-of-perceived contrast and highly inconsistent with perceived contrast. Albert's assertion is presumably based on his unjustified belief that the equality of these data indicate that observers are simply (or primarily) matching perceived contrast in the contrast troughs (and, moreover, that perceived contrasts in these local regions must be equal because their Michelson contrasts are approximately equal). The data do not support this view. First, Figure P1 Panel B clearly shows that perceived contrast and transmittance matches for the LCS differ by a fixed scaling factor over the entire curve, as our ratio-of-perceived contrast model predicts. Second, Albert's argument is based on his belief that the equality between normalized perceived contrast and transmittance data would not hold if one were to perform the equivalent perceived contrast experiment for our HCS. This is based on a flawed intuition because all models under consideration (perceived contrast, ratio-of-perceived contrast, or Albert's mixed model) predict that transmittance matches for the HCS must be identical to their perceived contrast matches. If observers use perceived contrast for their transmittance matches, they are equal by definition; if observers use ratio-of-perceived contrast, they are also equal because the transmittance anchor (hence normalization factor) for the HCS has a perceived contrast of $\sim 1$ (more precisely, 0.95 ). The perceived contrast and ratio-of- perceived contrast models can only be distinguished on the basis of our LCS, which clearly supports the ratio-of-perceived contrast model (Figure P1 Panel B). Thus, all of the data are fully consistent with our ratio-of-perceived contrast model, and deviate substantially from any model that involves simply (or even primarily) matching perceived contrasts.

Space restrictions allow only brief responses to Albert's (2008) other replies: Albert asserted that his data are largely consistent with the perceived contrast theory, which is inconsistent with his claim that observers could not perform contrast matches to his $\mathrm{f}=$ 0 pattern. Albert's claim that Robilotto and Zaidi's (2004) data contradict our ratio-of-perceived contrast model is unjustified, because they did not measure perceived transparency and perceived contrast using the same matching pattern. Observers' contrast and transparency data were therefore obtained on different scales, precluding a direct comparison with our model (something that is also true for Albert's data). Kasrai and Kingdom's (2001) data were very well fit by our ratio of contrasts model, as they noted in their article. In sum, Albert's arguments fail because the ratio-of-perceived contrast model can only be assessed with experiments in which perceived transmittance and perceived contrast are measured using the same match pattern and mapped onto a common scale, something that is missing in both Albert's experiments and the data he attempted to levy against our model from other articles. It also underlies the misunderstanding that leads him to claim that we are "quietly bringing back" a model we have previously rejected, as all of our data were mapped onto Michelson contrast for all of our experiments.

\section{References}

Albert, M. K. (2008). Postscript: Qualitative and quantitative processes in the perception of achromatic transparency. Psychological Review, 115, $1141-1143$.

Anderson, B. L., Singh, M., \& Meng, J. (2006). The perceived transmittance of inhomogeneous surfaces and media. Vision Research, 46, 1982 1995.

Kasrai, R., \& Kingdom, F. A. A. (2001). Precision, accuracy, and range of perceived achromatic transparency. Journal of the Optical Society of America, A, 18, 1-11.

Robilotto, R., \& Zaidi, Q. (2004). Perceived transparency of neutral density filters across dissimilar backgrounds. Journal of Vision, 4(3), 183-195. 